\title{
Follow-up of the manganese-exposed workers healthy cohort (MEWHC) and biobank management from 2011 to 2017 in China
}

Yanting Zhou ${ }^{1 \dagger}$, Xiaoting Ge ${ }^{1 \dagger}$, Yuefei Shen ${ }^{2}$, Lian Qin ${ }^{3}$, Yaoqiu Zhong ${ }^{1}$, Chao Jiang ${ }^{1}$, Cheng Su' ${ }^{1}$, Jinyu Huang ${ }^{3}$, Suzhen Lin ${ }^{3}$, Defu Li ${ }^{1}$, Hong Cheng ${ }^{1}$, Fu Wei ${ }^{4}$, Songfeng Ou ${ }^{4}$, Yunfeng Zou ${ }^{4,5}$ and Xiaobo Yang ${ }^{1,5,6^{*}}$

\begin{abstract}
Background: Long-term excess exposure to environmental manganese (Mn) can lead to multi-system damage, especially in occupational populations. Therefore, we established a manganese-exposed workers healthy cohort (MEWHC), focusing on the systemic health effects related to Mn exposure. Here, we aimed to describe the followup activity for the MEWHC study and establish a standardized biological sample bank for the scientific management of high-quality biospecimens and the attached data from 2011 to 2017.

Methods: Baseline examinations for onsite workers were conducted, and the biobank for the MEWHC was first established in 2011; follow-up examinations occurred four times between July 2012 and November 2017. All questionnaires, clinical data and biological samples were routinely collected during each follow-up activity. Additional workers were recruited in 2016, which further enriched the resources of the biobank.

Results: A total of 2359 onsite workers and 612 retired workers at a ferromanganese refinery were enrolled in the prospective cohort, and their biological samples were obtained in the preliminary baseline survey and the follow-up investigation, including 2971 blood and urine samples from the cohort. In addition, 1524 hair samples, 1404 nail (toe and finger nails) and 1226 fecal samples were also collected. All specimens were preserved in the biobank, and the data were scientifically managed using a computer system.

Conclusions: The MEWHC study in China provides an effective way to obtain biological samples such as plasma, DNA, hair and urine for storage in a biobank for further study. The standardized management of various samples is crucial for accessing high-quality biospecimens.
\end{abstract}

Keywords: Manganese-exposed workers healthy cohort, Biobank, Follow-up

\footnotetext{
* Correspondence: yxbo21021@163.com

†Yanting Zhou and Xiaoting Ge contributed equally to this work.

'Department of Occupational Health and Environmental Health, School of Public Health, Guangxi Medical University, Shuangyong Road 22, Nanning 530021, China

${ }^{5}$ Guangxi Colleges and Universities Key Laboratory of Prevention and Control of Highly Prevalent Diseases, Guangxi Medical University, Shuangyong Road 22, Nanning 530021, China

Full list of author information is available at the end of the article
}

(c) The Author(s). 2018 Open Access This article is distributed under the terms of the Creative Commons Attribution 4.0 International License (http://creativecommons.org/licenses/by/4.0/), which permits unrestricted use, distribution, and reproduction in any medium, provided you give appropriate credit to the original author(s) and the source, provide a link to the Creative Commons license, and indicate if changes were made. The Creative Commons Public Domain Dedication waiver (http://creativecommons.org/publicdomain/zero/1.0/) applies to the data made available in this article, unless otherwise stated. 


\section{Background}

The element manganese $(\mathrm{Mn})$ is essential for normal physiological functions in the human body. However, excessive Mn exposure can be toxic to the human body, causing particular harm to the central nervous system $[1,2]$. Humans are primarily exposed to Mn through their diet; however, another major route of exposure is via inhalation [3-5]. Overexposure may occur in occupational settings, especially from $\mathrm{Mn}$ mining dusts and welding fumes [6,7]. Previous studies have shown that workers exposed to welding fumes are much more likely to experience extrapyramidal, Parkinsonian-type movement disorder than matched controls [8]. Furthermore, people residing near factories that utilize $\mathrm{Mn}$ in production are also threatened [9].

In recent years, prospective and retrospective methods have been applied to explore the effects of $\mathrm{Mn}$ exposure on various organs. However, the exact mechanism by which Mn exerts toxicity in humans has not yet been revealed. Thus, to explore Mn-induced toxicity in the human body, especially regarding the systemic health effects of Mn exposure, we established a prospective Mn-exposed worker healthy cohort (MEWHC), which began in 2011 in China [10]. To date, investigations of the subjects in the cohort have included numerous aspects related to the early health effects of Mn exposure, such as lifestyle habits and socio-economic status as well as environmental, occupational and genetic factors. Furthermore, we have also collected a range of biological samples, including blood, urine, hair, and nails, to explore early health effects, potential biomarkers of exposure, disease, susceptibility and diseases related to occupational Mn exposure.

Biobanks form a chain of operations that include informing subjects and obtaining proper consent (depending on local requirements), data acquisition, biospecimen collection, annotation, preservation, storage, quality control, cataloguing, managing of access, processing and distribution [11]. They have gradually begun to play an increasingly important role in studying the determinants of population health and shaping proper prevention programs and health policies. Therefore, it is necessary to establish a standardized biobank to preserve and manage specimens for ongoing research. Consequently, the MEWHC biobank will provide researchers with a reliable way to access high-quality biospecimens with clinical data for basic scientific research and validation studies. In addition, specimen resources, such as serum, plasma and human tissues, are limited and valuable, which requires us to manage and use these samples and their related data reasonably and effectively.

\section{Methods}

\section{MEWHC study design and follow-up}

The prospective cohort study and the biobank for Mn-exposed workers began simultaneously in 2011. Health examinations of the participants are conducted annually. In our long-term follow-up survey, an increasing number of participants continue to be recruited into this heavy metal cohort, and the corresponding biobank and epidemiological data have been further enriched. The participants recruited into the cohort participate in annual follow-ups, and a comprehensive follow-up visit for all subjects is conducted every 3 years. As shown in Fig. 1, the biobank was established through the MEWHC study. The health examinations, questionnaires, files and environmental monitoring of the workers were conducted at baseline or via follow-up surveys. The collection of biological samples and related information includes a range of clinical, epidemiological and biological data, with integrated analysis by researchers.

\section{Standard protocol approvals, patient consent and investigator training}

All study protocols were reviewed and approved by the Medical Ethics Committee of Guangxi Medical University. All participants provided written consent to participate prior to the start of the study. Workers exposed to $\mathrm{Mn}$ were recruited from one large ferro-Mn alloy production plant in Guangxi, China. All of the subjects had worked in the ferromanganese refinery for at least 1 year and lived in the local area.

All investigators were trained in how to obtain and process biological samples. These instructions covered the importance of specimens, sample information recording, sample processing, packaging, labeling, sample transportation and preservation. Focusing efforts on stringent quality control and quality assurance measures in establishing the biobank are important first steps [12]. The investigator must focus on not only collecting enough specimens but also ensuring that the collected specimens are of the necessary quality. Moreover, the executives of the biobank require a certain level of experimental skill and management ability to ensure the realization of the entire process, ranging from sample collection to detection data feedback.

\section{Sample collection}

All individuals from the MEWHC were informed about health examinations, questionnaire investigations and donating biological samples. The most important step in the workflow of biospecimen acquisition and preservation is that the researchers must strictly comply with the rules of operation. They must ensure that the timing of all operations is consistent with both the requirements of optimal collection and the preservation of biological products, meaning that the sample storage conditions were comparable to other large-scale biobanks. Detailed information on biological specimen collection is presented as a flowchart in Fig. 2. 


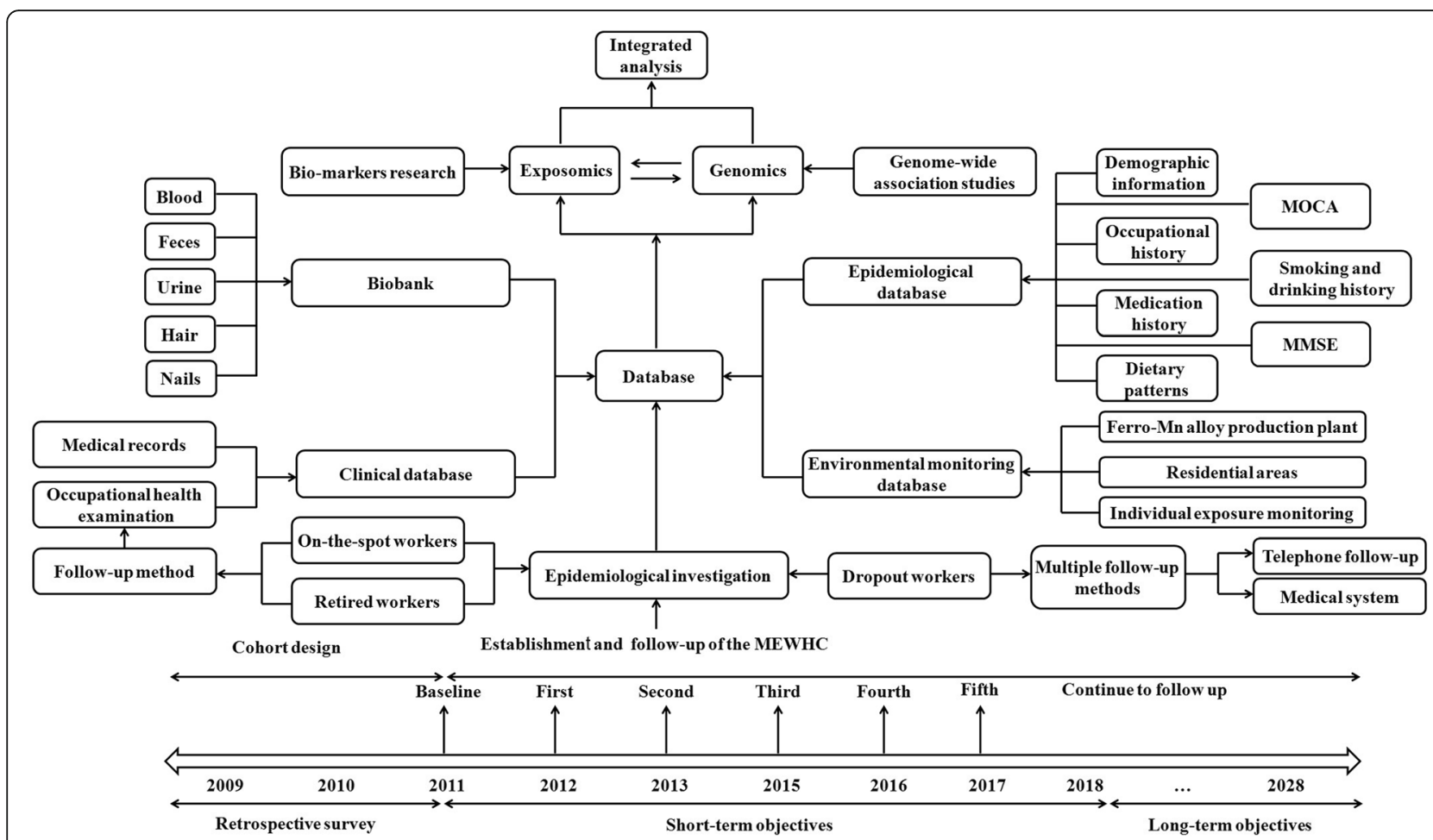

Fig. 1 Study plan of the manganese-exposed workers healthy cohort (MEWHC). MoCA: The Montreal Cognitive Assessment; MMSE: Mini-mental State Examination

\section{Collection of blood specimens and DNA extraction}

Blood samples were collected from workers with different cumulative exposure indexes (CEIs) from the 8-h air $\mathrm{Mn}$ time-weighted average (TWA) in the occupational environment. At the first investigation and during the follow-up visits every 2 years, $5 \mathrm{~mL}$ of peripheral venous blood was collected in EDTA tubes. The samples were centrifuged using a high-speed benchtop centrifuge (Thermo Fisher, Biofuge Stratos) to separate plasma, serum and blood cells, and they were then stored at $-80{ }^{\circ} \mathrm{C}$ (Thermo Fisher, U.S.A). We extracted DNA from blood collected in EDTA tubes using Takara Universal Genomic DNA Extraction kits based on the manufacturer's protocol (Takara, Japan).

\section{Collection of nail and hair specimens}

A sufficient quantity of nail samples was available for metal assays. The nail clippings were collected from the study participants who had an adequate toenail length, including all 10 toenails and 10 fingernails. Before sample processing and testing, all nail specimens were placed in small polyethylene bags, stored in a clean container with desiccant and then placed in a $-20{ }^{\circ} \mathrm{C}$ freezer (KK25V61TI, 254 L, Haier) in the laboratory. The nail clippings were analyzed as previously described in Kile et al., in 2007 [13]: they were sonicated for $15 \mathrm{~min}$ in $10 \mathrm{~mL}$ of $1 \%$ Triton $\mathrm{X}-100$ solution, rinsed with distilled deionized water, dried at $60{ }^{\circ} \mathrm{C}$ in a drying oven for $24 \mathrm{~h}$, weighed, digested in nitric acid and analyzed using inductively coupled plasma mass spectrometry (ICP-MS) (Nex10N350x, Agilent, USA).

Hair specimens were taken on the same day as other samples. Approximately $80 \mathrm{mg}$ of hair was taken from the nape of the head as close as possible to the scalp with stainless-steel scissors and stored in polyethylene bags. Hair samples were stored in the same way as nail samples before they were sent for trace element analysis. According to the modified method developed by the International Atomic Energy Agency [14], the hair specimen was cut into pieces, washed in deionized water, rinsed with acetone and then dried in an oven at $50 \pm$ $10{ }^{\circ} \mathrm{C}$. After drying, the hair samples were digested with nitric acid and then diluted with deionized water containing $1 \%$ nitric acid. Finally, the hair samples were analyzed by inductively coupled plasma-mass spectrometry (ICP-MS) (Nex10N350x, Agilent, USA).

\section{Collection of urine and fecal specimens}

A midstream urine sample $(>30 \mathrm{~mL})$ was collected in a plastic container, acidified to $1 \%(v / v) \mathrm{HNO}_{3}$, divided into aliquots and subsequently stored in polyethylene tubes at $-20{ }^{\circ} \mathrm{C}$ until shipment to Guangxi Medical University, where samples were stored frozen $\left(-80{ }^{\circ} \mathrm{C}\right.$, Thermo Fisher, USA) prior to further determination of $\mathrm{Mn}$ and trace elements levels. 


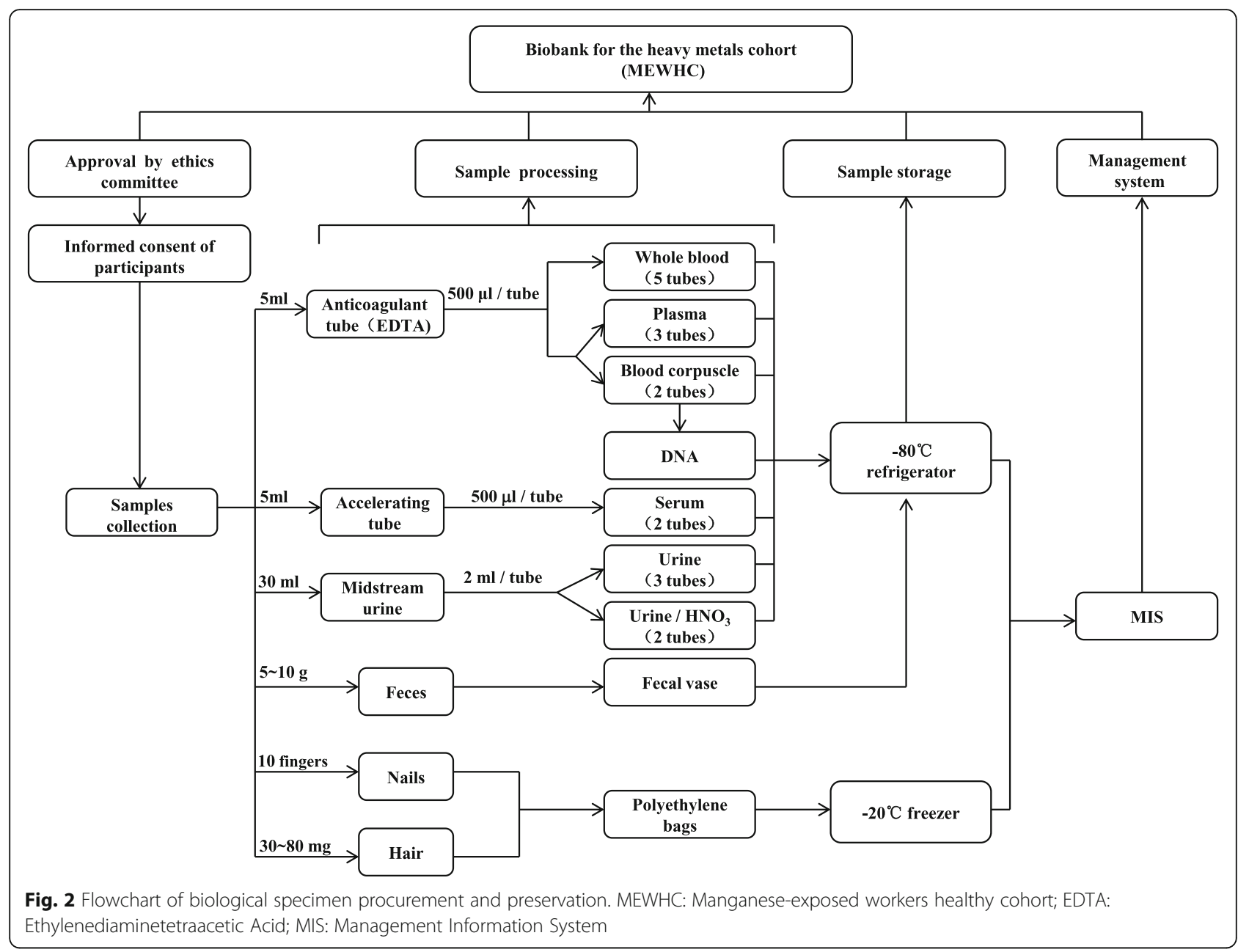

We started the collection of fecal samples in 2016. Accounting for the feasibility of on-site fecal specimen collection, including time limitations and the participants' compliance, we decided to collect the fecal specimens from retired ferroalloy plant workers. Following enrollment, participants were invited to provide a fresh fecal specimen. Immediately following collection, the samples were placed in a sterile feces tube containing no solution, and then the study investigators sealed the feces tubes and froze each specimen $\left(-80^{\circ} \mathrm{C}\right.$, Thermo Fisher, USA). The average time from sample collection to storage was less than $2 \mathrm{~h}$.

\section{Data collection}

Data collection included questionnaires, information on environmental exposure and clinical data.

The questionnaire collected information on demographics, socio-economic status, smoking history, alcohol consumption, medication history and occupational history. Smoking habits were classified as smoker (smoking at least 1 cigarette daily for more than 3 months) or non-smoker (never smoked or a former smoking habit with cessation for more than 3 months). Drinking habits were classified as current drinker (drinking at least once each week for more than 3 months) or nondrinker (never drank or formerly drank) [15]. In addition, 2 neurological function tests were conducted to assess and screen for the effects of $\mathrm{Mn}$ exposure on the nervous system using the following methods: the Montreal Cognitive Assessment (MoCA), a neurocognitive function test that rapidly screens for mild cognitive impairment with high sensitivity and specificity [16-19]; and the mini-mental state examination (MMSE), a scale for screening dementia that can comprehensively, accurately and rapidly reflect the intelligence status and cognitive impairment degree of the subjects [17, 19-21]).

The data on environmental exposure were divided into approximately 2 parts, including harmful occupational factors in the factory and the levels of $\mathrm{Mn}$ in the air in the residential area. We recorded the production processes and conditions of potential Mn pollution in this plant and detected the individual levels of Mn using individual samplers. The method used has been described in detail in previous studies [22]. We then calculated the cumulative exposure index (CEI) from the 8-h air $\mathrm{Mn}$ 
TWA in each department of the factory and multiplied it by the Mn exposure-years for each worker. In particular, we performed more comprehensive air monitoring through personal samplers according to the participants' occupations in 2017. This method enabled the exploration of accurate exposure levels among the participants in different occupations. More detailed information on personal samplers according to their occupations has been described in (Additional file 1: Table S1). Information about the jobs and tasks of the subjects has been described in detail in previous studies [10].

Clinical data were obtained from medical records, which were matched to the stored specimens during every epidemiological investigation. Baseline and follow-up surveys were conducted face-to face or via telephone interviews. Then, all of the available data were recorded and stored in information databases or the biobank.

\section{Biobank management system}

A biobank management system mainly includes a hardware facility system, a monitoring system (temperature monitoring) and an information management system. The hardware facility system was equipped with an electronic system and refrigeration and included a $4{ }^{\circ} \mathrm{C}$ refrigerator, a $-20{ }^{\circ} \mathrm{C}$ freezer, a $-80{ }^{\circ} \mathrm{C}$ low-temperature freezer and $\mathrm{a}-152^{\circ} \mathrm{C}$ liquid nitrogen tank. The management system, as mentioned above, was used to manage all biological samples and related data, especially the detailed records of the participants' exposure history in each follow-up activity, which was controlled by professionally trained staff. Secure data storage and high-quality digital preservation and annotation of information related to the biosamples are indispensable. One staff member was trained to supervise collecting and inputting all clinical information.

Each subject has a unique identifier (Barcode printer, B-2404, TSC, China), which can facilitate linking different databases and helps researchers to conveniently manage biospecimens. Furthermore, there are strict rules for the use of samples, which must be recorded in detail for any experiment on the samples and under the supervision of the bank manager. In that case, biobank users must be able to fully track samples in interoperable electronic systems that pay particular attention to the quality and security of the data. More information can be found in Additional file 2.

\section{Statistical analysis}

A Chi-squared test was used to perform statistical analyses of the frequency data. The significance level for all statistical tests was 0.05 . All statistical analyses were performed using the software package SPSS (Version 21.0).

\section{Results}

Demographic characteristics of the cohort

As shown in Table 1, the basic characteristics of 2971 workers included retired workers $(n=612)$ and onsite workers $(n=2359)$. The retired worker group consisted of 231 men (37.75\%) and 381 women (62.25\%), and the onsite worker group consisted of 1453 men (61.59\%) and 906 women (38.41\%). The average age of the retired workers was 69.7 years, and that of the onsite workers was 40.0 years. Of the retired workers, $63(10.29 \%)$ were current smokers, and $132(21.57 \%)$ were current drinkers. Of the onsite workers, 877 (37.18\%) were current smokers, and 1071 (45.40\%) were current drinkers, and the current passive smoking rates were 46.92 and $85.67 \%$, respectively. Based on the biobank for the heavy metal cohort, except for the laboratory tests, which included routine blood tests, routine urine tests and liver function tests, we detected levels of $\mathrm{Mn}$ and $\mathrm{Fe}$ in blood, urine or hair using atomic absorption spectrometry. In this case, we combined the environmental exposures provided at the level of each cohort member with other individual-level data and biobank samples for further epidemiological analysis.

\section{Establishment of the biobank}

During the 6-year active period of this prospective longitudinal study, 2359 onsite workers and 612 retired workers were enrolled in the heavy metal cohort for occupational health examination, and series of different types of biological samples were obtained, including blood and urine samples of each participants, 1226 fecal samples, 1404 nail samples and 1524 hair samples. The results of the sample types and all of the numbers of biosamples collected from 2011 to 2017 are shown in (Additional file 3:Table S2). In addition, we monitored the occupational hazardous factors in the working environment of the research objectives, especially the level of Mn dust in the air.

\section{Findings based on the MEWHC study}

Currently, the data show that working with ferroalloy is related to reduced lung ventilation function. Furthermore, the MEWHC study also showed that Mn exposure is related to decreased lung ventilation function in male smelter workers; individual smoking habits and $\mathrm{Mn}$ exposure have a synergistic effect on decreased lung function [23]. Liver function was compared among the different Mn-exposed groups in 2013, and we found that occupational Mn exposure can cause a dose-dependent increase in liver enzyme levels and can interact with alcohol consumption to aggravate liver damage [10]. Regarding the biomarkers of exposure, disease and susceptibility, the levels of plasma brain-derived neurotrophic factor (BDNF) and dopamine were determined using sandwich 
Table 1 Demographic data of the study population

\begin{tabular}{|c|c|c|c|c|c|}
\hline \multirow[t]{3}{*}{ Variables } & \multicolumn{2}{|c|}{ Onsite workers } & \multicolumn{2}{|c|}{ Retired workers } & \multirow[t]{3}{*}{$P$} \\
\hline & Number & Percent & Number & Percent & \\
\hline & \multicolumn{2}{|l|}{$(n=2359)$} & \multicolumn{2}{|l|}{$(n=612)$} & \\
\hline \multicolumn{6}{|l|}{ Sex } \\
\hline Male & 1453 & 61.59 & 231 & 37.75 & 0.00 \\
\hline Female & 906 & 38.41 & 381 & 62.25 & \\
\hline \multicolumn{6}{|l|}{ Age, years } \\
\hline$<30$ & 249 & 10.56 & 0 & 0.00 & 0.00 \\
\hline $30-40$ & 818 & 34.68 & 0 & 0.00 & \\
\hline $40-50$ & 1100 & 46.63 & 4 & 0.65 & \\
\hline $50-60$ & 185 & 7.84 & 105 & 17.16 & \\
\hline $60-70$ & 7 & 0.30 & 159 & 25.98 & \\
\hline $70-80$ & 0 & 0.00 & 245 & 40.03 & \\
\hline$\geq 80$ & 0 & 0.00 & 99 & 16.18 & \\
\hline \multicolumn{6}{|l|}{ Race/ethnicity } \\
\hline Han Chinese & 1148 & 48.66 & 401 & 65.52 & 0.00 \\
\hline Zhuang minority & 1109 & 47.01 & 189 & 30.88 & \\
\hline Other ethnic groups & 92 & 3.90 & 21 & 3.43 & \\
\hline Missing & 10 & 0.42 & 1 & 0.16 & \\
\hline \multicolumn{6}{|l|}{ Marital status } \\
\hline Single & 293 & 12.42 & 11 & 1.80 & 0.00 \\
\hline Married & 1989 & 84.32 & 451 & 73.69 & \\
\hline Widowed or divorced & 73 & 3.09 & 149 & 24.35 & \\
\hline Missing & 4 & 0.17 & 1 & 0.16 & \\
\hline \multicolumn{6}{|l|}{ Education } \\
\hline Middle school or lower & 948 & 40.19 & 470 & 76.80 & 0.00 \\
\hline High school & 1050 & 44.51 & 111 & 18.14 & \\
\hline University/college or higher & 358 & 15.18 & 29 & 4.74 & \\
\hline Missing & 3 & 0.13 & 2 & 0.33 & \\
\hline \multicolumn{6}{|l|}{ Seniority, years } \\
\hline$<10$ & 775 & 32.85 & 8 & 1.31 & 0.00 \\
\hline $10-20$ & 750 & 31.79 & 90 & 14.71 & \\
\hline $20-30$ & 693 & 29.38 & 260 & 42.48 & \\
\hline$\geq 30$ & 138 & 5.85 & 253 & 41.34 & \\
\hline Missing & 3 & 0.13 & 1 & 0.16 & \\
\hline \multicolumn{6}{|l|}{ Smoking status } \\
\hline Current smoker & 877 & 37.18 & 63 & 10.29 & 0.00 \\
\hline Former smoker & 160 & 6.78 & 81 & 13.24 & \\
\hline Never smoker & 1317 & 55.83 & 466 & 76.14 & \\
\hline Missing & 5 & 0.21 & 2 & 0.33 & \\
\hline \multicolumn{6}{|l|}{ Drinking status } \\
\hline Current drinker & 1071 & 45.40 & 132 & 21.57 & 0.00 \\
\hline Former drinker & 386 & 16.36 & 102 & 16.67 & \\
\hline Never drinker & 896 & 37.98 & 374 & 61.11 & \\
\hline Missing & 6 & 0.25 & 4 & 0.65 & \\
\hline
\end{tabular}


Table 1 Demographic data of the study population (Continued)

\begin{tabular}{|c|c|c|c|c|c|}
\hline \multirow[t]{3}{*}{ Variables } & \multicolumn{2}{|c|}{ Onsite workers } & \multicolumn{2}{|c|}{ Retired workers } & \multirow[t]{3}{*}{$P$} \\
\hline & Number & Percent & Number & Percent & \\
\hline & \multicolumn{2}{|c|}{$(n=2359)$} & \multicolumn{2}{|l|}{$(n=612)$} & \\
\hline \multicolumn{5}{|l|}{$\mathrm{BMI}, \mathrm{kg} / \mathrm{m}^{2}$} & 0.00 \\
\hline$<18.5$ & 88 & 3.73 & 9 & 1.47 & \\
\hline $18.5-24$ & 1445 & 61.25 & 232 & 37.91 & \\
\hline $24-28$ & 670 & 28.40 & 251 & 41.01 & \\
\hline$\geq 28$ & 136 & 5.77 & 94 & 15.36 & \\
\hline Missing & 20 & 0.85 & 26 & 4.25 & \\
\hline Height, cm (mean \pm SD) & \multicolumn{2}{|c|}{$163.18 \pm 7.56$} & \multicolumn{2}{|c|}{$155.29 \pm 7.78$} & \\
\hline Weight, kg (mean \pm SD) & \multicolumn{2}{|c|}{$60.46 \pm 9.95$} & \multicolumn{2}{|c|}{$57.99 \pm 9.68$} & \\
\hline
\end{tabular}

$B M I$ body mass index. $S D$ standard deviation

ELISA kits (ChemiKine, USA), and the biomarkers of susceptibility were assessed using genome-wide association studies (GWAS) [10]. The results showed that a decrease in plasma BDNF levels and cognitive impairment may be caused by occupational Mn exposure.

\section{Discussion}

Banking biosamples from population-based cohorts holds significant research potential today, and the MEWHC biobank has been the core of the heavy metal cohort. The present biobank also offers an open research platform to provide an integral resource for biological samples, including blood, plasma, DNA and human tissues, which can be used for the discovery, validation and implementation of biomarkers or potential new biomarkers of exposure, disease and susceptibility.

Thus far, we have investigated a series of biomarkers based on the MEWHC biobank, especially biomarkers for exposure and susceptibility. Our results suggest that $\mathrm{Mn}$ in blood cells might serve as a biomarker of Mn exposure [24]. Larger validation studies using the rich biological biosamples of the MEWHC biobank are needed to determine the utility of this biomarker. Furthermore, our results show that Mn exposure may be associated with cognition impairment in this large cross-sectional study. There is evidence to suggest that higher serum BDNF levels are associated with better neuropsychological function [25]. Thus, the detection of biomarker BDNF was performed immediately, and animal and molecular biology experiments were also conducted to investigate the role of BDNF. A negative dose-response relationship between plasma BDNF and Mn exposure levels was observed. Therefore, plasma BDNF levels can be a potential marker of cognitive impairment induced by Mn exposure [22].

Since the establishment of the biobank, its researchers have been committed to the standardization of biospecimen management. The entire sample management process, including collection, transport, processing and storage, will be strengthened, especially in the following three aspects: preventing samples from being polluted by various biomolecules throughout the management process, from collection from the Mn-exposed workers to storage in the biobank; controlling time and temperature in sample transportation, both of which affect biospecimen quality; and establishing standard operating procedures for the centrifugation of blood, DNA extraction and anticorrosion of urine, among others.

We also focused on how to maximize the research usability of the biospecimens since the number of biospecimens is limited [26]. In the past, samples used for $\mathrm{Mn}$ analysis to explore associations between $\mathrm{Mn}$ exposure and concentrations in biosamples were examined using traditional atomic absorption spectrometry; however, we can now detect the concentrations of 22 metals using inductively coupled plasma mass spectrometry (ICP-MS). Experimenters should be required to optimize their use of biosamples to maximize opportunities for further research based on these biosamples [27]. At present, new technologies are becoming more refined so that even very small amounts of sample can become rich sources of discovery; in particular, the biospecimens from patients diagnosed with Mn poisoning, which are few and precious, will be a powerful resource in the Mn-exposed cohort to support mechanism research. Therefore, the dropout rate of the workers is still a limitation of the current research-the dropout rate of the MEWHC was $23.23 \%(548 / 2359)$. Due to the dynamic characteristics of the MEWHC, one of the largest challenges for specimen collection is reducing the dropout rate. In recent years, some workers have chosen to leave or transfer to civilian work for various reasons.

To address these challenges, we have primarily adopted 3 types of follow-up methods according to the reasons for the dropout. In the future, we will continue to organize and conduct occupational health examinations for onsite 
or retired workers. Additionally, we also telephone the subjects who are leaving the area or transferring to civilian work to obtain information, and even track outcomes, through the medical system.

In addition, the MEWHC biobank has made a significant contribution to the present research findings and provides rich sample resources for further research. It should be open to external researchers as a platform that allows scholars to search for and identify biospecimens in order to develop and validate new value-based biomarkers. The biobank aims to facilitate data sharing and pooling across multiple population-based biobanks of heavy metal cohorts to stimulate cooperation among investigators and to maximize the utility of biospecimens in research. Furthermore, the MEWHC biobank also provides an open channel to share the research findings generated using human biospecimens; therefore, it can contribute to many research projects.

\section{Conclusions}

The biosamples stored in the MEWHC biobank, such as plasma, DNA, hair and urine, will make important contributions toward the discovery and validation of biomarkers. Thus far, the MEWHC biobank has played important roles in epidemiology studies and in exploring the genetic susceptibility of Mn exposure. Further analyses of information from GWAS, combined with the results from molecular toxicology analyses, are crucial to facilitating our future studies. Therefore, the longitudinal MEWHC will be continued, and the standardization of the biobank management system and expansion of the specimen database are necessary.

\section{Additional files}

Additional file 1: Table S1. Air monitoring of different workshops and jobs in the ferro-Mn alloy production plant in 2017. (PDF $107 \mathrm{~kb}$ )

Additional file 2: Supplementary information for the biobank of the MEWHC. (PDF 398 kb)

Additional file 3: Table S2. Summary of the number of samples deposited in the biobank obtained from the heavy-metal cohort (MEWHC) initiated in 2011. (PDF $89 \mathrm{~kb}$ )

\section{Abbreviations \\ BDNF: Brain-derived Neurotrophic Factor; BMI: Body Mass Index; CEl: The Cumulative Exposure Index; EDTA: Ethylenediaminetetraacetic Acid; GWAS: Genome-wide Association Studies; ICP-MS: Inductively Coupled Plasma Mass Spectrometry; MEWHC: Manganese-exposed Workers Healthy Cohort; MIS: Management Information System; MMSE: Mini-mental State Examination; MoCA: The Montreal Cognitive Assessment; TWA: Time-weighted Average}

\section{Acknowledgements}

We thank all participants who volunteered to take part in this study, all researchers of the MEWHC research team and the nurses and administrators in the ferromanganese refinery.

\section{Funding}

This study was supported by the National Natural Science Foundation of China (81472962), the Guangxi Science Fund for Distinguished Young Scholars (2012GXNSFFA 060009) and the Fok Ying Tung Education Foundation (141118).

\section{Availability of data and materials}

The data and material are available upon reasonable request from the corresponding author. E-mail: yxbo21021@163.com.

\section{Authors' contributions}

XBY contributed to conception and design; XTG, YFS, LQ, YQZ, CJ, CS, JYH, SZL, DFL, HC, FW, SFO, YFZ and YTZ contributed to acquisition of the data; YTZ and XTG analyzed the data and drafted the manuscript. XTG and YTZ contributed equally to this study. All authors approved the final manuscript of this article prior to submission.

\section{Ethics approval and consent to participate}

The Medical Ethics Committee of Guangxi Medical University approved this study. All participants were fully informed about the study purpose and methods and provided written consent to participate.

\section{Consent for publication}

Not applicable.

\section{Competing interests}

The authors declare that they have no competing interests.

\section{Publisher's Note}

Springer Nature remains neutral with regard to jurisdictional claims in published maps and institutional affiliations.

\section{Author details}

${ }^{1}$ Department of Occupational Health and Environmental Health, School of Public Health, Guangxi Medical University, Shuangyong Road 22, Nanning 530021, China. ${ }^{2}$ Department of Neurology, The First Affiliated Hospital of Guangxi Medical University, Nanning, Guangxi, China. ${ }^{3}$ The First Clinical Medical College, Guangxi University of Science and Technology, Liuzhou, Guangxi, China. ${ }^{4}$ Department of Toxicology, School of Public Health, Guangxi Medical University, Nanning, Guangxi, China. ${ }^{5}$ Guangxi Colleges and Universities Key Laboratory of Prevention and Control of Highly Prevalent Diseases, Guangxi Medical University, Shuangyong Road 22, Nanning 530021, China. ${ }^{6}$ Center for Genomic and Personalized Medicine, Guangxi Medical University, Shuangyong Road 22, Nanning 530021, China.

Received: 28 March 2018 Accepted: 24 July 2018

Published online: 01 August 2018

\section{References}

1. Tuschl K, Clayton PT, Gospe SM Jr, Gulab S, Ibrahim S, Singhi P, et al. Syndrome of hepatic cirrhosis, dystonia, polycythemia, and hypermanganesemia caused by mutations in SLC30A10, a manganese transporter in man[J]. Am J Hum Genet. 2012;90(3):457-66.

2. Stanwood GD, Leitch DB, Savchenko V, Wu J, Fitsanakis VA, Anderson DJ, et al. Manganese exposure is cytotoxic and alters dopaminergic and GABAergic neurons within the basal ganglia[J]. J Neurochem. 2009;110(1): 378-89.

3. Williams M, Todd GD, Roney N, Crawford J, Coles C, McClure PR, et al. Agency for Toxic Substances and Disease Registry (ATSDR) Toxicological Profiles. In: Toxicological Profile for Manganese. Atlanta (GA): Agency for Toxic Substances and Disease Registry (US); 2012.

4. Chakraborty S, Martinez-Finley E, Caito S, Chen P, Aschner M. CHAPTER 9 Manganese. In: Garner D, editor. Binding, Transport and Storage of Metal lons in Biological Cells: The Royal Society of Chemistry; 2014. p. 260-81.

5. Lucchini RG, Guazzetti S, Zoni S, Donna F, Peter S, Zacco A, et al. Tremor, olfactory and motor changes in Italian adolescents exposed to historical ferro-manganese emission[J]. Neurotoxicology. 2012;33(4):687-96.

6. Racette BA, McGee-Minnich L, Moerlein SM, Mink JW, Videen TO, Perlmutter JS. Welding-related parkinsonism: clinical features, treatment, and pathophysiology[]]. Neurology. 2001;56(1):8-13. 
7. Sriram K, Lin GX, Jefferson AM, Roberts JR, Chapman RS, Chen BT, et al. Dopaminergic neurotoxicity following pulmonary exposure to manganesecontaining welding fumes[]]. Arch Toxicol. 2010;84(7):521-40.

8. Racette BA, Criswell SR, Lundin Jl, Hobson A, Seixas N, Kotzbauer PT, et al. Increased risk of parkinsonism associated with welding exposure[]]. Neurotoxicology. 2012;33(5):1356-61.

9. Chen P, Culbreth M, Aschner M. Exposure, epidemiology, and mechanism of the environmental toxicant manganese[J]. Environ Sci Pollut Res Int. 2016; 23(14):13802-10

10. Lv Y, Zou Y, Liu J, Chen K, Huang D, Shen Y, et al. Rationale, design and baseline results of the Guangxi manganese-exposed workers healthy cohort (GXMEWHC) study[J]. BMJ Open. 2014;4(7):e005070.

11. Hainaut P, Caboux E, Bevilacqua G, Bosman F, Dassesse $T$, Hoefler $H$, et al. Pathology as the cornerstone of human tissue banking: European consensus expert group report[J]. Biopreservation and Biobanking. 2009;7(3): 157-60

12. Betsou F, Barnes R, Burke T, Coppola D, Desouza Y, Eliason J, et al. Human biospecimen research: experimental protocol and quality control tools[J]. Cancer Epidemiol Biomarkers Prev. 2009;18(4):1017-25.

13. Kile ML, Houseman EA, Breton CV, Quamruzzaman Q, Rahman M, Mahiuddin $G$, et al. Association between total ingested arsenic and toenail arsenic concentrations[]]. J Environ Sci Health A Tox Hazard Subst Environ Eng. 2007;42(12):1827-34.

14. Puchyr RF, Bass DA, Gajewski R, Calvin M, Marquardt W, Urek K, et al. Preparation of hair for measurement of elements by inductively coupled plasma-mass spectrometry (ICP-MS)[J]. Biol Trace Elem Res. 1998;62(3):167-82.

15. Deng Q, Liu J, Li Q, Chen K, Liu Z, Shen Y, et al. Interaction of occupational manganese exposure and alcohol drinking aggravates the increase of liver enzyme concentrations from a cross-sectional study in China[]]. Environ Health. 2013;12:30

16. Nasreddine ZS, Phillips NA, Bedirian V, Charbonneau S, Whitehead V, Collin I. et al. The Montreal cognitive assessment, MoCA: a brief screening tool for mild cognitive impairment[J]. J Am Geriatr Soc. 2005:53(4):695-9.

17. Fisekovic S, Memic A, Pasalic A. Correlation between Moca and mmse for the assessment of cognition in schizophrenia[J]. Acta informatica medica: AIM : I Society for Med Informatics of Bosnia \& Herzegovina : casopis Drustva za medicinsku informatiku BiH. 2012:20(3):186-9.

18. Freitas S, Simoes MR, Alves L, Duro D, Santana I. Montreal cognitive assessment (MoCA): validation study for frontotemporal dementia[J]. J Geriatr Psychiatry Neurol. 2012;25(3):146-54

19. Kim JI, Sunwoo MK, Sohn YH, Lee PH, Hong JY. The MMSE and MoCA for screening cognitive impairment in less educated patients with Parkinson's disease[J]. J Mov Disord. 2016;9(3):152-9.

20. Qiao J, Zheng X, Wang X, Lu W, Cao H, Qin X. Neuropsychological profile in Chinese patients with Parkinson's disease and normal global cognition according to mini-mental state examination score[J]. Int J Clin Exp Med. 2015;8(8):13755-61.

21. Li H, Jia J, Yang Z. Mini-mental state examination in elderly Chinese: a population-based normative study[J]. J Alzheimer's Dis. 2016;53(2):487-96.

22. Zou Y, Qing L, Zeng X, Shen Y, Zhong Y, Liu J, et al. Cognitive function and plasma BDNF levels among manganese-exposed smelters[]]. Occup Environ Med. 2014;71(3):189-94.

23. Wang F, Zou Y, Shen Y, Zhong Y, LV Y, Huang D, et al. Synergistic impaired effect between smoking and manganese dust exposure on pulmonary ventilation function in Guangxi manganese-exposed workers healthy cohort (GXMEWHC)[J]. PLoS One. 2015;10(2):e0116558.

24. Ge X, Wang F, Zhong Y, Lv Y, Jiang C, Zhou Y, et al. Manganese in blood cells as an exposure biomarker in manganese-exposed workers healthy cohort[J]. J Trace Elem Med Biol. 2018:45:41-7.

25. Gunstad J, Benitez A, Smith J, Glickman E, Spitznagel MB, Alexander T, et al. Serum brain-derived neurotrophic factor is associated with cognitive function in healthy older adults[]]. J Geriatr Psychiatry Neurol. 2008;21(3): 166-70.

26. Riegman PH, de Jong B, Daidone MG, Soderstrom T, Thompson J, Hall JA, et al. Optimizing sharing of hospital biobank samples[J]. Sci Transl Med. 2015; 7(297):297fs31

27. Schneider D, Riegman PH, Cronin M, Negrouk A, Moch H, Balling R, et al. Accelerating the development and validation of new value-based diagnostics by leveraging biobanks[]]. Public Health Genomics. 2016;19(3): $160-9$.

\section{Ready to submit your research? Choose BMC and benefit from:}

- fast, convenient online submission

- thorough peer review by experienced researchers in your field

- rapid publication on acceptance

- support for research data, including large and complex data types

- gold Open Access which fosters wider collaboration and increased citations

- maximum visibility for your research: over $100 \mathrm{M}$ website views per year

At BMC, research is always in progress.

Learn more biomedcentral.com/submissions 